\title{
Individual differences in dealing with incomplete information: Judging clinical competence
}

\author{
IRWIN P. LEVIN \\ University of Iowa, Iowa City, Iowa \\ RICHARD D. JOHNSON \\ University of Alberta, Edmonton, Alberta, Canada \\ and \\ DANIEL P. CHAPMAN \\ University of Iowa Hospitals and Clinics, Iowa City, Iowa
}

\begin{abstract}
Recent research on human judgment and decision making has shown that subjects can use their perception of the relation between stimulus dimensions to infer or impute a value for missing information. Considerable individual differences have been evident. The present study tested the hypothesis that individual differences in the magnitude of the inference effect are related to the weight or importance attached to the missing information and the presumed nature of the interdimensional relationship. The hypothesis was tested in a task in which subjects were asked to rate the competence of hypothetical clinical psychologists on the basis of the clinicians' experience in treating patients with a particular problem and their familiarity with recent developments in treatment.
\end{abstract}

How do decision makers cope with situations in which information about some factor that is important to the decision or judgment is not readily available? Three strategies have been suggested and tested. First, they may search for the missing information, particularly if the information is important and the cost of the search is relatively low (e.g., Meyer, 1981). Second, they may ignore the factor for which information is missing. This assumption is implicit in studies that attempt to identify informationintegration rules by means of varying the amount of information available across trials (e.g., Anderson, 1965). Finally, they may use the information available for other factors to infer a value for the missing factor (Huber \& McCann, 1982; Johnson, 1988; Levin, Johnson, \& Faraone, 1984; Singh, 1991; Yamagishi \& Hill, 1981).

The tendency to infer the value of missing information from the relation between stimulus dimensions and incorporate inferred values into the evaluation appears to vary both between subjects (Levin et al., 1984) and between information dimensions within subjects (Singh, 1991). The present study further explores individual differences in dealing with missing information and provides new evidence for imputations of missing values in a task involving judgments of clinical competence.

The task required judgments of clinical competence based on information about prior experience in treating patients and degree of familiarity with recent develop-

The authors would like to thank Patricia Deldin and Craig Russo for their assistance with this project. Correspondence should be addressed to Irwin P. Levin, Department of Psychology, University of Iowa, Iowa City, IA 52242. ments in treatment. A scenario was established for perceiving these two factors as negatively related, because this provides the best opportunity for distinguishing between common information-integration effects and the effects of imputing values to missing information.

\section{METHOD}

\section{Stimulus Design}

The subjects were asked to rate each of a series of hypothetical clinical psychologists in terms of their competence for treating the problem of severe depression, resulting from loneliness, in college students. The clinical psychologists were described by the factors number of patients seen and familiarity with recent developments in treatment. The levels of each factor were specified as much above average, above average, average, below average, or much below average.

Instead of forming all possible pairs of values of the two stimulus factors, a design representative of a possible negative relation between factors was selected. Clinicians were described as average, above average, or much above average on one factor and much below average, below average, or average on the other factor. This results in two $3 \times 3$ factorial subdesigns, as illustrated in Figure 1. (Note that the stimulus pair average number of patients-average familiarity appears in each subdesign, resulting in a total of 17 distinct stimulus pairs.) In addition, the subjects were given single-stimulus descriptions consisting of numberof-patients-only trials (with no information about familiarity with recent developments) and familiarity-only trials (with no information about number of patients seen). Ten such single-stimulus trials were included, representing each level of each factor.

\section{Subjects}

The subjects were 62 students from introductory psychology classes at the University of Iowa.

\section{Procedure}

The subjects were provided a cover story suggesting a negative relationship between stimulus factors. They were told that recent advancements in treating this kind of depression will be much more familiar to recent graduates than to long-time practitioners; conversely, long-time 


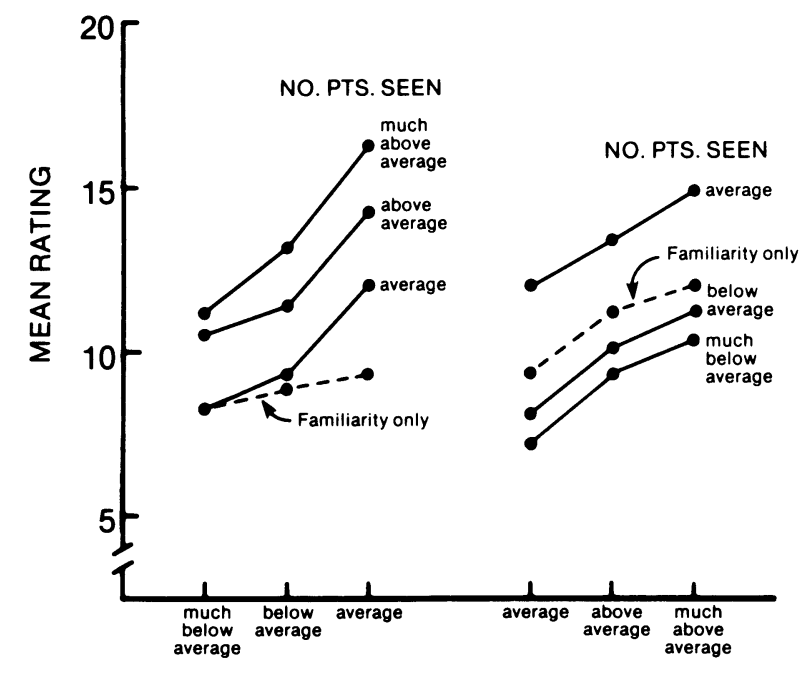

FAMILIARITY

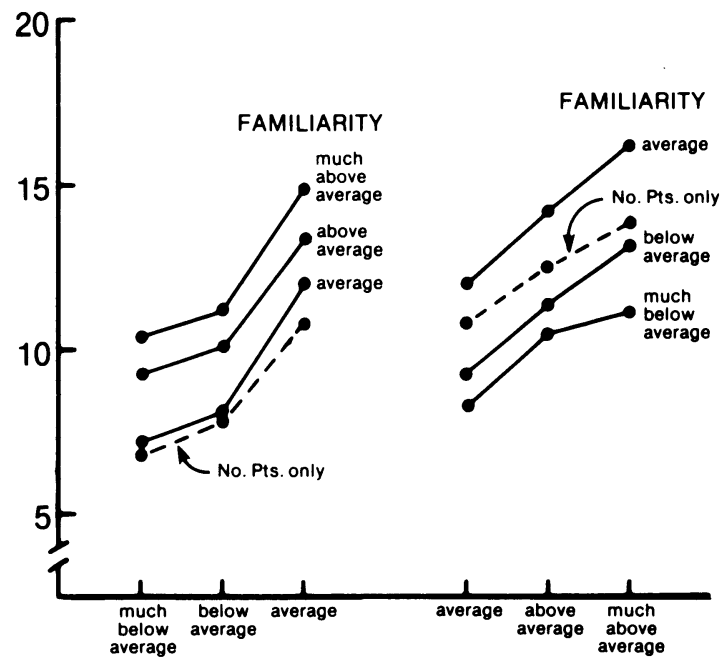

NO. PTS. SEEN

\section{SUBJECTS WHO RATED "FAMILIARITY" AS MORE IMPORTANT}
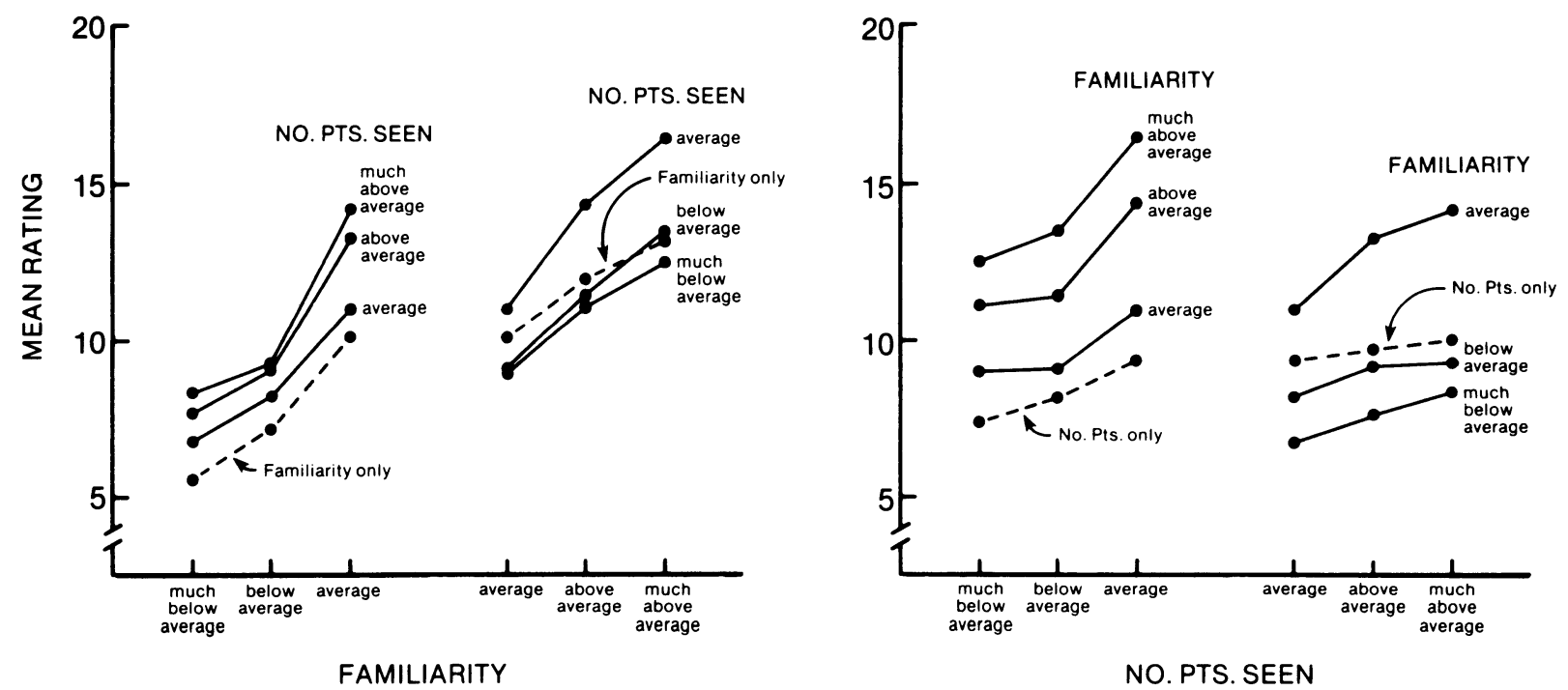

Figure 1. Mean ratings as a function of number of patients seen ("No. Pts. Seen") and familiarity with recent developments ("Familiarity") for the subjects who perceived a negative relationship between factors. The subjects were classified in terms of which factor they rated as more important.

practitioners will have had more experience dealing with the problem than will the recent graduates. Thus, some clinicians will be well informed about recent developments in treatment but will not have had much actual clinical experience, while others will have had considerable clinical experience but will not be very familiar with the most recent developments.

The subjects were given a booklet containing two replications of the complete design (the 17 stimulus-pair trials and the 10 single-stimulus trials). A different random order of presentation of the 27 descriptions was provided for each replication. The subjects were told that each description in the booklet represented a different hypothetical clinical psychologist. They were asked to rate each clinician in terms of how well qualified they thought that clinician was for treating lonely, depressed college students. They were told to use a scale of 1 to 20 , where the more qualified they judged a clinician to be, the higher the number they should assign.

\section{Importance Ratings and Relationship Check}

Following completion of their ratings, the subjects were asked the following question: "In making your judgments of competency, which factor did you consider to be more important-number of patients seen or $f a-$ miliarity with recent development?' Responses to this question were used to classify the subjects on the basis of their weighting of the two factors.

Finally, the subjects were given the task of filling in values of missing information when only one factor was presented. Each level of number of patients seen and each level of familiarity with recent develop- 


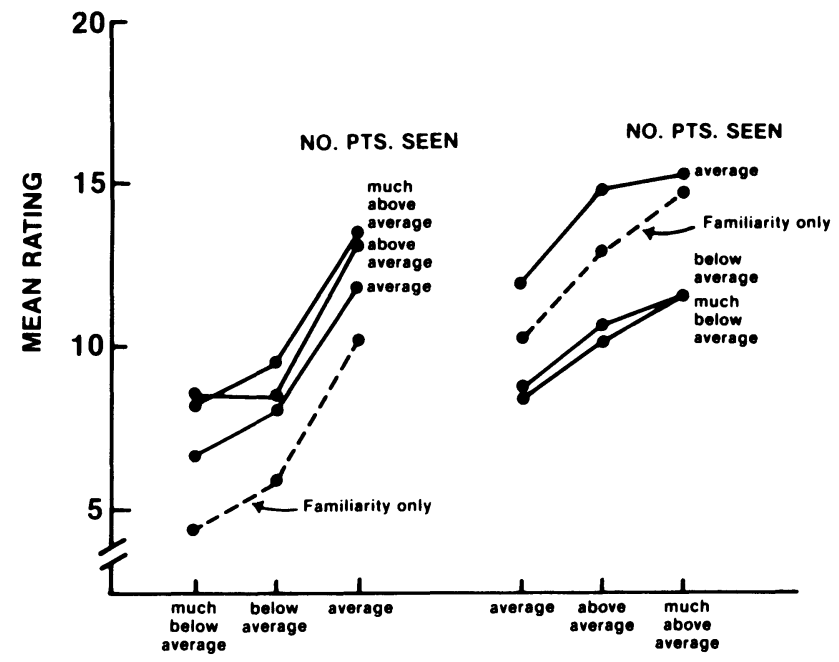

FAMILIARITY

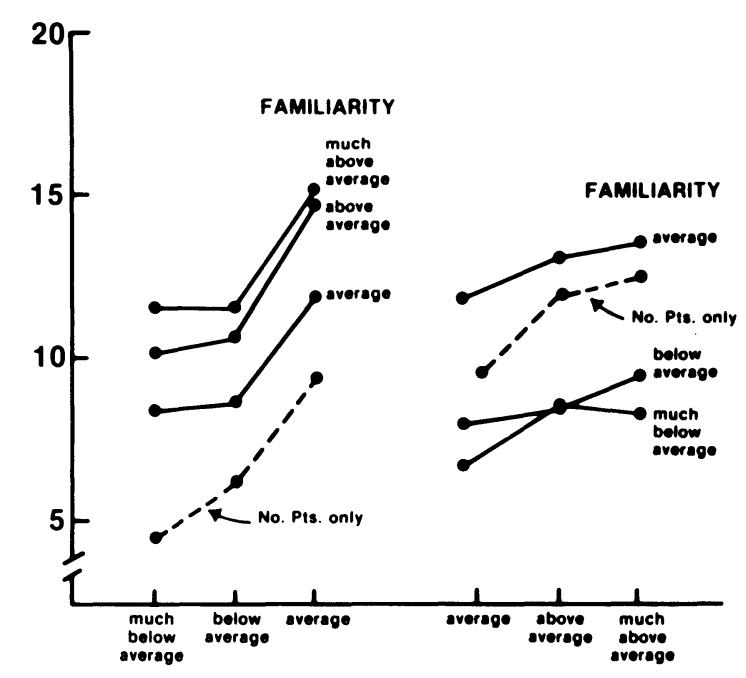

NO. PTS. SEEN

Figure 2. Mean ratings as a function of number of patients seen and familiarity with recent developments for the subjects who perceived a positive relationship between factors.

ments were presented in random order. The subjects were instructed to fill in the blanks by giving what they thought would be the average value of the missing factor for the given level of the presented factor. These "explicit inferences" were used to determine which subjects actually perceived a negative relation between the two factors.

\section{RESULTS}

For purposes of examining individual differences in imputing values to missing information, the subjects were classified in terms of their explicit inference responses and their designation of the more important factor in their judgments. Forty-two out of the 62 subjects apparently did perceive a negative relation between the factors. That is, when asked to fill in values of missing information, they tended to indicate below-average values for one factor when the other factor was above average, and aboveaverage values when the other factor was below average. Of these, 22 subjects indicated that number of patients seen was the more important factor, and 20 subjects indicated that familiarity with recent developments was the more important factor. On the basis of explicit inferences, 20 subjects apparently perceived a positive relation between the factors; number of patients seen was the more important factor for 5 of these subjects, and familiarity was the more important factor for 15 subjects.

Results for the subjects who perceived a negative interstimulus relation are plotted in Figure 1. Each panel plots data from two subdesigns: low levels of familiarity with recent developments paired with high levels of number of patients seen, and high levels of familiarity paired with low levels of number of patients seen. The upper panels plot the data for the subjects who indicated number of patients seen as the more important factor; the bottom panels plot the data for the subjects who indicated familiarity with recent developments as the more important factor. The left panels plot mean ratings for stimulus pairs (solid lines) and mean ratings for single-stimulus trials with number of patients seen as the missing factor (dotted lines). The right panels plot mean ratings for stimulus pairs (solid lines, based on the same data as in the left panels, but with a different variable on the abscissa) and mean ratings for single-stimulus trials with familiarity with recent developments as the missing factor (dotted lines). Results for the subjects who perceived a positive interstimulus relation are shown in Figure 2. Because of low sample size; these data are collapsed over factor seen as more important.

A finding common to all panels in Figures 1 and 2 is that the dotted line falls between the solid lines for average and below average. This shows that our subjects tended to give lower than average ratings when one source of information was missing, suggesting that the fact that information was missing was perceived somewhat negatively (see also Johnson \& Levin, 1985; Meyer, 1981; Yates, Jagacinski, \& Faber, 1978).

More important for present concerns are the differences between figures. The most obvious difference between Figures 1 and 2 is the relative slopes of the solid and dotted lines. In Figure 1, the dotted lines are flatter than or parallel to the solid lines. In Figure 2, the dotted lines tend to be steeper than the solid lines. In each subdesign of each panel of the figures, the difference in slope between the dotted line and the (pooled) solid lines varies significantly between the subjects who perceived a negative interstimulus relation and those who perceived a positive relation ( $F$ values ranged from 4.64 to $18.05 ; p<.05$, in each case). This provides strong evidence that subjects use imputed values of missing information in their evaluation on single-stimulus trials and that these imputations are mediated by individual differences in perception of the relation between the two stimulus dimensions.

Evidence for the role of individual differences in estimation of the more important factor can be found by comparing the various panels in Figure 1. The subjects' selfreports of which factor was more important were related 
to how they weighted the factors in the judgment task. The magnitude of the effect of manipulating number of patients seen corresponds to the degree of separation of the solid lines in the left panels. The magnitude of the effect of manipulating familiarity with recent developments corresponds to the degree of separation of the solid lines in the right panels. The significantly wider spread between the solid lines in the upper left panel than between those in the lower left panel shows that the subjects who reported greater weight for number of patients seen did in fact give greater weight to this factor than did the subjects who reported greater weight for familiarity with recent developments. The lower panels show corresponding results for the subjects who reported greater weight for familiarity with recent developments.

Evidence that the subjects used a perceived negative relation between factors to infer missing values occurs when the effect of a given factor is reduced on single-stimulus trials relative to that on stimulus-pair trials. This is because the effect of a given factor is reduced or "neutralized" when subjects infer an opposite value for the other factor. This would lead to flatter slopes for the dotted lines depicting responses on single-stimulus trials than for the solid lines depicting responses to stimulus pairs. Evidence that subjects impute a constant value to missing information on a given stimulus dimension can be seen when the dotted line is parallel to the solid lines. The observation that the dotted lines tend to be flatter than or parallel to the solid lines in Figure 1 is in direct contrast to results predicted by the traditional averaging model of information integration, which assumes that a stimulus factor has its greatest effect when it is not combined with another factor. This prediction translates into steeper slopes for the dotted lines than for the solid lines. (The assumption that imputations are based on a presumed positive relation between factors leads to the same prediction.)

The tendency for the dotted line to be flatter than the solid lines is somewhat more pronounced in the left subdesign of the upper left panel and the right subdesign of the lower right panel. Because the upper left panel and lower right panel represent the data where the most important source of information for a given subject is missing, such a result would support the hypothesis that the inference effect based on a perceived negative relation is greater when the missing values are from the subjectively more important source of information. The effect, however, is rather small. The dotted line is significantly flatter than the (pooled) solid lines in three of the four attribute/ subdesign tests (combined over self-reported weight), but the degree of difference between solid and dotted lines is significantly related to the self-reported weight for only one of the four tests [left subdesign of left panel; $F(1,40)=$ $5.36, p<.05]$.

\section{DISCUSSION}

This study confirms earlier findings that when people are faced with a judgment or decision for which not all of the key information is available, they may impute a value to the missing information. In the present case, a scenario was established for perceiving a negative relation between factors. Evidence for imputing values to missing information was especially clear for the subjects who in fact perceived there to be a negative relation between number of patients seen and familiarity with recent developments. In such cases, the usual finding in support of the averaging model of information integration-that the effect of variations in a stimulus dimension is greatest when no additional information is presented-is absent or even reversed.

Individual differences in imputing values to missing information were evident when comparing the subjects who perceived a negative interdimensional relationship with those who perceived a positive relation. The difference in slopes of the dotted lines in Figures 1 and 2 is consistent with the assumption that imputations of missing information values were mediated by the perceived relation between presented and missing sources of information. The mediating role of perceived importance of the two stimulus dimensions was more equivocal. There was only suggestive evidence that the magnitude of the inference effect was related to the weight of the missing source of information. Given the subjects' relative unfamiliarity with the evaluation of clinical psychologists, a priori differences in presumed importance may not have been as effective as those differences produced by manipulation of weight, as in Johnson's (1988) study, which provided stronger support for the relation between weight and inference effects.

\section{REFERENCES}

ANDERSON, N. H. (1965). Averaging versus adding as a stimulus combination rule in impression formation. Journal of Experimental Psychology, 70, 394-400.

Huber, J., \& MCCANN, J. (1982). The impact of inferential beliefs on product evaluations. Journal of Marketing Research, 19, 324-333.

JoHnson, R. D. (1988). Making decisions with incomplete information: The first complete test of the inference model. In T. Srull (Ed.), Advances in consumer research (Vol. 16, pp. 522-528). Provo, UT: Association for Consumer Research.

Johnson, R. D., Levin, I. P. (1985). More than meets the eye: The effect of missing information on purchase evaluations. Journal of Consumer Research, 12, 169-177.

Levin, I. P., Johnson, R. D., \& FARAone, S. V. (1984). Information integration in price-quality tradeoffs: The effects of missing information. Memory \& Cognition, 12, 96-102.

MeYer, R. J. (1981). A model of multiattribute judgments under attribute uncertainty and informational constraint. Journal of Marketing Research, 18, 428-441.

SINGH, R. (1991). Two problems in cognitive algebra: Imputations and averaging-versus-multiplying. In N. H. Anderson (Ed.), Contributions to information integration theory: Vol. II. Social (pp. 143-180). Hillsdale, NJ: Erlbaum.

Yamagishi, T., \& HiLl, C. T. (1981). Adding versus averaging models revisited: A test of a path-analytic integration model. Journal of Personality \& Social Psychology, 41, 13-25.

YATES, J. F., JAGACINSKI, C. J., \& FABER, M. D. (1978). Evaluation of partially described multi-attribute options. Organizational Behavior \& Human Performance, 21, 240-251.

(Manuscript received April 27, 1991.) 